\title{
Physiologic upper limit of pore size in the blood-tumor barrier of
} malignant solid tumors

\author{
Hemant Sarin*1,2, Ariel S Kanevsky², Haitao Wu ${ }^{3}$, Alioscka A Sousa ${ }^{1}$, \\ Colin M Wilson ${ }^{3}$, Maria A Aronova ${ }^{1}$, Gary L Griffiths ${ }^{3}$, Richard D Leapman ${ }^{1}$ \\ and Howard Q $\mathrm{Vo}^{1,2}$
}

\begin{abstract}
Address: ${ }^{1}$ National Institute of Biomedical Imaging and Bioengineering, National Institutes of Health, Bethesda, Maryland 20892, USA, ${ }^{2}$ Radiology and Imaging Sciences Program, Clinical Center, National Institutes of Health, Bethesda, Maryland 20892, USA and ${ }^{3}$ Imaging Probe Development Center, National Heart, Lung, and Blood Institute, National Institutes of Health, Bethesda, Maryland 20892, USA

Email: Hemant Sarin* - sarinh@mail.nih.gov; Ariel S Kanevsky - kanevskya@mail.nih.gov; Haitao Wu - wuh3@mail.nih.gov; Alioscka A Sousa - sousaali@mail.nih.gov; Colin M Wilson - wilsoncm@mail.nih.gov; Maria A Aronova - aronovaa@mail.nih.gov; Gary L Griffiths - griffithsgl@mail.nih.gov; Richard D Leapman - leapmanr@mail.nih.gov; Howard Q Vo - voho@mail.nih.gov

* Corresponding author
\end{abstract}

Published: 23 June 2009

Journal of Translational Medicine 2009, 7:5 I doi:10.| | 866/|479-5876-7-5 I
Received: 27 April 2009

Accepted: 23 June 2009

This article is available from: http://www.translational-medicine.com/content/7/I/5 I

(c) 2009 Sarin et al; licensee BioMed Central Ltd.

This is an Open Access article distributed under the terms of the Creative Commons Attribution License (http://creativecommons.org/licenses/by/2.0), which permits unrestricted use, distribution, and reproduction in any medium, provided the original work is properly cited.

\begin{abstract}
Background: The existence of large pores in the blood-tumor barrier (BTB) of malignant solid tumor microvasculature makes the blood-tumor barrier more permeable to macromolecules than the endothelial barrier of most normal tissue microvasculature. The BTB of malignant solid tumors growing outside the brain, in peripheral tissues, is more permeable than that of similar tumors growing inside the brain. This has been previously attributed to the larger anatomic sizes of the pores within the BTB of peripheral tumors. Since in the physiological state in vivo a fibrous glycocalyx layer coats the pores of the BTB, it is possible that the effective physiologic pore size in the BTB of brain tumors and peripheral tumors is similar. If this were the case, then the higher permeability of the BTB of peripheral tumor would be attributable to the presence of a greater number of pores in the BTB of peripheral tumors. In this study, we probed in vivo the upper limit of pore size in the BTB of rodent malignant gliomas grown inside the brain, the orthotopic site, as well as outside the brain in temporalis skeletal muscle, the ectopic site.
\end{abstract}

Methods: Generation 5 (G5) through generation 8 (G8) polyamidoamine dendrimers were labeled with gadolinium (Gd)-diethyltriaminepentaacetic acid, an anionic MRI contrast agent. The respective Gd-dendrimer generations were visualized in vitro by scanning transmission electron microscopy. Following intravenous infusion of the respective Gd-dendrimer generations (Gd-G5, $N=6 ; G d-G 6, N=6 ; G d-G 7, N=5 ; G d-G 8, N=5$ ) the blood and tumor tissue pharmacokinetics of the Gd-dendrimer generations were visualized in vivo over 600 to 700 minutes by dynamic contrast-enhanced MRI. One additional animal was imaged in each Gd-dendrimer generation group for 175 minutes under continuous anesthesia for the creation of voxel-by-voxel Gd concentration maps.

Results: The estimated diameters of Gd-G7 dendrimers were $1 \mathrm{I} \pm 1 \mathrm{~nm}$ and those of Gd-G8 dendrimers were $13 \pm I \mathrm{~nm}$. The BTB of ectopic RG-2 gliomas was more permeable than the BTB 
of orthotopic RG-2 gliomas to all Gd-dendrimer generations except for Gd-G8. The BTB of both ectopic RG-2 gliomas and orthotopic RG-2 gliomas was not permeable to Gd-G8 dendrimers.

Conclusion: The physiologic upper limit of pore size in the BTB of malignant solid tumor microvasculature is approximately $I 2$ nanometers. In the physiologic state in vivo the luminal fibrous glycocalyx of the BTB of malignant brain tumor and peripheral tumors is the primary impediment to the effective transvascular transport of particles across the BTB of malignant solid tumor microvasculature independent of tumor host site. The higher permeability of malignant peripheral tumor microvasculature to macromolecules smaller than approximately $12 \mathrm{~nm}$ in diameter is attributable to the presence of a greater number of pores underlying the glycocalyx of the BTB of malignant peripheral tumor microvasculature.

\section{Background}

The blood-tumor barrier (BTB) of malignant solid tumor microvasculature is more permeable to macromolecules than the endothelial barrier of normal tissue microvasculature of the continuous type[1,2]. This hyper-permeability of malignant solid tumor microvasculature to macromolecules has been attributed to the local release of vascular permeability factor in tumor tissue $[3,4]$. The BTB of malignant solid tumors growing outside the brain in peripheral tissues and organs is typically more permeable than the BTB of similar malignant solid tumors growing in the brain $[5,6]$. Furthermore, when a malignant peripheral tumor, such as a breast cancer tumor, metastasizes to the brain, an ectopic site, the permeability of the BTB of the breast cancer tumor growing in the brain is lower than the BTB of the original tumor in breast tissue, the orthotopic site[5]. The brain tissue host site microenvironment lowers the permeability of the BTB of metastatic malignant peripheral tumors such that it approximates the permeability of the BTB of orthotopic brain tumors like malignant gliomas[7,8].

Various sizes of pores have been identified in the BTB of malignant solid tumor microvasculature, which is discontinuous[1]. These include trans-endothelial cell fenestrations, caveolae and vesiculo-vacuolar organelles (VVOs) within endothelial cells, and inter-endothelial cell gaps between endothelial cells[1,4,9-12]. Based on electron microscopy, the anatomic pore size of the fenestrations, caveolae, and VVOs of the BTB of both brain tumors and peripheral tumors have been reported to range between $40 \mathrm{~nm}$ and $200 \mathrm{~nm}$ in diameter[10,13,14]. In contrast, the pore size of inter-endothelial cell gaps within the BTB of both brain tumors and peripheral tumors is much larger. In the case of brain tumors, inter-endothelial cell gaps have been reported to range between $100 \mathrm{~nm}$ and 3000 $\mathrm{nm}$ in diameter $[10,13]$ and in the case of peripheral tumors the gaps have been reported to range between 300 $\mathrm{nm}$ and $4700 \mathrm{~nm}[12]$. Although the diameters of the trans-endothelial cell fenestrations, caveolae, and VVOs are smaller than those of the inter-endothelial cell gaps, these pores are more numerous than the inter-endothelial cell gaps in the BTB of brain tumors and peripheral tumors $[4,9,10]$. The higher permeability of the BTB of peripheral tumors compared to the BTB of brain tumors has been previously attributed to the presence of larger inter-endothelial gaps in the BTB of peripheral tumors[12,15].

The pore size within the BTB of malignant solid tumors has been previously probed in vivo with intra-vital microscopy after the intravenous infusion of particles in the nanometer size range labeled on the exterior with rhodamine, a cationic fluorescent dye $[15,16]$. Cationic particles are known to be toxic to the negatively charged glycocalyx $[17,18]$, which is the fibrous carbohydrate layer that coats the luminal surface of endothelial cells[19]. As a result cationic particles have been shown to increase the permeability of the BTB by disrupting the glycocalyx of the BTB [20-22]. With intra-vital fluorescence microscopy the transvascular extravasation of cationic nanoparticles across the BTB of malignant tumor microvasculature has been visualized and it has been reported that the upper limit of pore size within the BTB of malignant brain tumors ranges between $7 \mathrm{~nm}$ and $100 \mathrm{~nm}$, whereas that the upper limit of pore size within the BTB of peripheral tumors ranges between $200 \mathrm{~nm}$ and $1200 \mathrm{~nm}$ [15].

In the case of malignant brain tumors, we recently probed the upper limit of pore size within the BTB of orthotopic RG-2 rat gliomas with dynamic contrast-enhanced MRI using dendrimer nanoparticles labeled on the exterior with gadolinium (Gd)-diethyltriaminepentaacetic acid (DTPA), an anionic MRI contrast agent[22]. Based on this work, we reported that the upper limit of pore size within the BTB of orthotopic RG-2 rat gliomas in vivo was approximately $12 \mathrm{~nm}[22]$. These previously reported findings suggest that the impediment to the transvascular extravasation of particles across the BTB of brain tumors is at the level of the glycocalyx that coats the surface of the pores in the BTB and is a "nanofilter" for the transvascular flow of particles across the BTB[23]. 
It is possible that the physiologic upper limit of pore size within the BTB of peripheral tumors previously reported as being between $200 \mathrm{~nm}$ and $1200 \mathrm{~nm}[15]$ may be a gross over-estimation of the actual physiologic upper limit of pore size within the BTB of peripheral solid tumors. Therefore, if the actual physiologic upper limit of pore size within the BTB of peripheral tumors is significantly lower than what has been previously reported, and approximates that of the BTB of brain tumors, then this finding would suggest that more pores in BTB of peripheral tumors are the primary reason for the higher permeability of the BTB of malignant peripheral tumors compared to that of malignant brain tumors. Furthermore, such findings would have important implications on the size range of therapeutics that could be effectively delivered across the BTB of malignant solid tumors independent of tumor host site.

In our previous dynamic contrast-enhanced MRI-based work[22], we had characterized the upper limit of pore size within the BTB of orthotopic RG-2 malignant gliomas using successively higher generation $(\mathrm{G})$ polyamidoamine (PAMAM) dendrimers labeled with Gd-DTPA. With dynamic-contrast enhanced MRI, we found there to be significant positive contrast enhancement of brain tumor tissue following the intravenous infusion of Gd-G1 through Gd-G7 dendrimers, but not following the intravenous infusion of Gd-G8 dendrimers. Based on this observation, we established that Gd-G8 dendrimers were larger than the physiologic upper limit of pore size within the BTB of orthotopic RG-2 gliomas. With this dynamic contrast-enhanced MRI approach, in addition to being able to image the tumor tissue pharmacokinetics of GdG1 through Gd-G8 dendrimers, we were also able to image at the same time the blood pharmacokinetics of the respective Gd-dendrimer generations in the large vessels within the brain. We found that the higher generation GdG5 through Gd-G8 dendrimers maintained steady state blood concentrations over the 120 minute long imaging session. Since Gd-G5, Gd-G6, and Gd-G7 dendrimers maintained steady state blood concentrations over the 120 minute imaging session and were permeable to the BTB of orthotopic RG-2 brain tumors, these higher generation Gd-dendrimers continued to accumulate within the tumor tissue extravascular space over time, and remained there for sufficiently long to localize within individual glioma tumor cells. Although these imaging sessions were long enough to determine the physiologic upper limit of pore size in the BTB of orthotopic brain tumors as well as qualitatively assess the blood half-lives of lower generation Gd-dendrimers, we were unable to qualitatively assess the blood half-lives of the higher generation Gddendrimers, since the higher generation Gd-dendrimers maintained steady state blood concentrations over 120 minutes.
In present study, we imaged the blood and tumor tissue pharmacokinetics of higher generation Gd-dendrimers over 600 to 700 minutes in order to characterize the differences in the permeability of the BTB of orthotopic and ectopic RG-2 malignant gliomas and define the upper limit of pore size within the BTB of brain tumors and peripheral tumors. We determined the differences in the permeability of the BTB of an ectopic RG-2 glioma and an orthotopic RG-2 glioma within the same rat at the same time. For each animal, RG-2 glioma cells were inoculated in the right anterior brain, which was the orthotopic site, and the left temporalis muscle, which was the ectopic site. The change in blood and tumor tissue Gd concentration, a surrogate for the Gd-dendrimer concentration, was determined by calculating the molar relaxivity of the respective Gd-dendrimer generation in vitro, and the change in the longitudinal relaxation time before and after Gd-dendrimer bolus for each imaged volume element (voxel) in vivo over time.

\section{Methods}

PAMAM dendrimer functionalization and characterization Bifunctional chelating agents and functionalized gadolinium-benzyl-diethyltriaminepentaacetic acid (Gd-BzDTPA) PAMAM dendrimers were synthesized according to procedures previously described[22]. With a molar reactant ratio of $=2: 1$ bifunctional chelate to dendrimer surface amine groups, isothiocyanate activated DTPA was reacted with the amine groups for 48 hours. Gadolinium was then chelated after the removal of the $t$-butyl protective groups on the DTPA. The percent by mass of Gd in each Gd-dendrimer generation was determined by elemental analysis to be: Gd-G5 (13.2\%), Gd-G6 (13.0\%), Gd-G7 (12.3\%), and Gd-G8 (11.9\%). Gd-G5 and Gd-G6 dendrimer molecular weights were determined by matrix assisted laser desorption/ionization time-of-flight (MALDI TOF) mass spectroscopy (Scripps Center for Mass Spectrometry, La Jolla, CA). Gd percent by mass of the Gddendrimer, in its solid form, was determined with the inductively coupled plasma-atomic emission spectroscopy (ICP-AES) method (Desert Analytics, Tucson, AZ). Gd-dendrimer infusions were normalized to $100 \mathrm{mM}$ with respect to Gd.

\section{In vitro scanning transmission electron microscopy}

For in vitro transmission electron microscopy (TEM) experiments, a $5 \mu \mathrm{L}$ droplet of phosphate-buffer saline solution containing a sample of either Gd-G5, Gd-G6, Gd-G7 or Gd-G8 dendrimers was adsorbed onto a $3 \mathrm{~nm}$ thick carbon support film covering lacey carbon electron microscopy grids. After adsorption for 2 minutes, the grids were blotted with filter paper to remove excess solution, washed 5 times with $5 \mu \mathrm{L}$ aliquots of deionized water, and left to dry in air. Annular dark-field (ADF) scanning transmission electron microscopy (STEM) images of the Gd- 
dendrimers were recorded using a Tecnai TF30 electron microscope (FEI, Hillsboro, OR, USA) equipped with a Schottky field-emission gun and an in-column ADF detector (Fischione, Export, PA, USA). Molecular weight measurements of Gd-G7 and Gd-G8 dendrimers were performed with a combination STEM and energy-filtered TEM (EFTEM) imaging approach $[24,25]$.

\section{In vitro magnetic resonance imaging for calculations of Gd-dendrimer molar relaxivity}

From each of the Gd-dendrimer stock solutions to be used for in vivo imaging, $20 \mu \mathrm{L}$ of Gd-dendrimer was withdrawn and diluted in $200 \mu \mathrm{L}$ microfuge tubes containing PBS. The final concentrations of each Gd-dendrimer generation were $0.00 \mathrm{mM}, 0.25 \mathrm{mM}, 0.50 \mathrm{mM}, 0.75 \mathrm{mM}$ and $1.00 \mathrm{mM}$ concentrations with respect to $\mathrm{Gd}$. As an external control, Magnevist (Bayer, Toronto, Canada), a form of Gd-DTPA, was also diluted in $200 \mu \mathrm{L}$ microfuge tubes containing PBS at the above concentrations. The microfuge tubes were secured in level and upright positions within a plastic container filled with deionized ultra pure water. The container was placed in a $7 \mathrm{~cm}$ small animal solenoid radiofrequency coil (Philips Research Laboratories, Hamburg, Germany), which was then centered within a 3.0 tesla MRI scanner (Philips Intera; Philips Medical Systems, Andover, MA). Gd signal intensity measurements were made using a series of $T_{1}$ weighted spin echo sequences with identical $T_{\mathrm{E}}$ (echo time, $10 \mathrm{~ms}$ ) but different $T_{\mathrm{R}}$ (repetition times; $100 \mathrm{~ms}, 300 \mathrm{~ms}, 600 \mathrm{~ms}$, and $1200 \mathrm{~ms}$ ). Using the measured Gd signal intensities and known $T_{\mathrm{R}}$ and $T_{\mathrm{E}}$ values, the equilibrium magnetization $\left(\mathrm{M}_{0}\right)$ and the longitudinal relaxivity $\left(1 / T_{1}\right)$ values were determined by non-linear regression (Eq. 1)[26].

$$
S=M_{0}\left(1-\exp \left(-\frac{T_{R}}{T_{1}}\right)\right) \exp \left(-\frac{T_{E}}{T_{2}}\right)
$$

The Gd-dendrimer molar relaxivities $\left(r_{1}\right)$ was calculated by linear regression (Eq. 2)[26].

$$
\frac{1}{T_{1}}=\frac{1}{T_{10}}+r_{1}[G d]
$$

The in vitro and in vivo Gd-dendrimer molar relaxivities were assumed to be equivalent for the purposes of this work[27].

\section{Orthotopic and ectopic RG-2 glioma induction and animal preparation for imaging}

All animal experiments were approved by the National Institutes of Health Clinical Center Animal Care and Use Committee. Cryofrozen pathogen-free RG-2 glioma cells were obtained from the American Type Culture Collection (Rockville, MD) and cultured in sterile DME supplemented with $10 \%$ FBS and $2 \%$ penicillin-streptomycin in an incubator set at $37^{\circ} \mathrm{C}$ and $5 \% \mathrm{CO}_{2}$. The anesthesia route for all animal experiments was isoflurane by inhalation with nose cone, $5 \%$ for induction and 1 to $2 \%$ for maintenance. On experimental day 0 , the head of anesthetized adult male Fischer344 rats (F344) weighing 190 to 200 grams (Harlan Laboratories, Indianapolis, IN) was secured in a stereotactic frame with ear bars (David Kopf Instruments, Tujunga, CA). The right brain caudate nucleus (orthotopic RG-2 glioma)[28] and left temporalis muscle (ectopic RG-2 glioma) locations were stereotactically inoculated with $10^{5} \mathrm{RG}-2$ glioma cells in $5 \mu \mathrm{L}$ of sterile PBS. In each location, the cells were injected over 8 minutes, using a $10 \mu \mathrm{L}$ Hamilton syringe with a blunt tip 32-gauge needle for the brain inoculate and a sharp tip 26-gauage needle for the temporalis muscle inoculate. On experimental days 11 to 12 , brain imaging of re-anesthetized rats was performed following placement of polyethylene femoral venous cannula (PE-50; Becton-Dickinson, Franklin Lakes, NJ) for contrast agent infusion. Gd-dendrimers were infused at dose of $0.09 \mathrm{mmol} \mathrm{Gd} / \mathrm{kg}$.

\section{In vitro magnetic resonance imaging of RG-2 gliomas}

For imaging, the animal was positioned supine, with face, head, and neck snugly inserted into a nose cone within the $7 \mathrm{~cm}$ small animal solenoid radiofrequency coil, which was then centered within the 3.0 tesla MRI scanner. Coronal, sagittal, and axial localizer scans were used in order to identify the coronal plane most perpendicular to the rat brain dorsum. After orienting the rat brain in the image volume, a fast spin echo $T_{2}$ weighted anatomical scan was performed. Image acquisition parameters for the $T_{2}$ scan were: $T_{\mathrm{R}}$ of $6000 \mathrm{~ms}, T_{\mathrm{E}}$ of $70 \mathrm{~ms}$, image matrix of 256 by 256, and slice thickness of $1 \mathrm{~mm}$. In order to quantify contrast agent concentration during post imaging processing, two separate three-dimensional fast field echo $T_{1}$ weighted scans were performed, one at a $3^{\circ}$ low flip angle (low FA) of and the other at a $12^{\circ}$ high flip angle (high FA). Image acquisition parameters for both scans were: $T_{\mathrm{R}}$ of $8.1 \mathrm{~ms}, T_{\mathrm{E}}$ of $2.3 \mathrm{~ms}$, image matrix of 256 by 256, and slice thickness of $1 \mathrm{~mm}$. The low FA scan was performed over $1.67 \mathrm{~min}$, without any Gd-dendrimer on board. For the high FA scans, which were the dynamic scans, the entire brain volume was acquired once every 20 seconds.

At the beginning of the first high FA scan, three to five precontrast brain volumes were acquired to guarantee the integrity of the $T_{1}$ map without contrast agent $\left(T_{10}\right)$. Following acquisition of the pre-contrast brain volumes, a $0.09 \mathrm{mmol} / \mathrm{kg}$ dose of the respective Gd-dendrimer generation was infused. The Gd-dendrimer was infused as a slow bolus, over 1 minute, so that the blood pharmacokinetics of the respective Gd-dendrimer generation could be accurately measured during the early time points. The initial series of high FA dynamic scans were acquired for 15 minutes and subsequent high FA dynamic scans were acquired over 2 minutes at various time points. For each 
of the imaging sessions to acquire the Gd signal intensity data for measurement of the change in blood and tumor tissue Gd concentration over 600 to 700 minutes, the rat brains of 2 to 3 rats were imaged as frequently as possible one after the other, once every 30 to 90 minutes. For each of subsequent high FA dynamic scan, the animal was reanesthetized and re-imaged. For each of the Gd-dendrimer generations, one additional rat head was imaged every 10 min following the initial 15 minute dynamic scan, for a total of 175 minutes, while the animal was maintained under anesthesia for the duration of the scanning session. This was to image more frequently the change in Gd signal intensity and produce voxel-by-voxel Gd concentration maps.

\section{Dynamic contrast-enhanced MRI data processing and analysis}

Imaging data was analyzed using the Analysis of Functional NeuroImaging (AFNI; http://afni.nimh.nih.gov/) software suite[29]. Motion correction was performed by registering each volume of the high FA dynamic scans to the low FA scan. After volume registration, a $T_{1}$ without contrast $\left(T_{10}\right)$ map was generated for each voxel by using the low FA signal data and the mean of the high FA dynamic scan signal data before contrast enhancement from the Gd-dendrimer bolus was visualized on the high FA dynamic scan (Eq. 3)[26].

$$
S_{10}=\frac{M_{0}\left(1-E_{10}\right) \sin \theta}{1-E_{10} \cos \theta} \text { where } E_{10}=\exp \left(-\frac{T_{R}}{T_{10}}\right)
$$

After generating the $T_{10}$ map, a $T_{1}$ map was generated for each voxel of each dynamic image of each high FA dynamic scan data set after the contrast enhancement. For the high FA scan data of the 2 minute scan sessions, the average Gd signal intensity data from the 6 dynamic scans was used for the $T_{1}$ map calculation. Using the $T_{10}$ and $T_{1}$ signal intensity map values, in addition to the Gd-dendrimer molar relaxivity value, each Gd signal data set was converted to a Gd concentration space data set (Eq. 2).

To determine the Gd concentration in the blood and RG2 gliomas, blood and tumor voxels, respectively, were selected on coronal images of the high FA dynamic scan data sets. The Gd concentration in blood was determined in the common carotid arteries, since these were the largest caliber brain vessels in the imaging field-of-view. From within the common carotid arteries, 5 to 10 voxels that had physiologically reasonable blood $T_{10}$ values of approximately $1100 \mathrm{~ms}$ were selected. To determine the change in blood Gd concentration over time the selected blood voxels were identified on the co-registered high FA dynamic scan data sets of the subsequent time points. The average blood Gd concentration values were then calculated for each time point.

To determine the Gd concentration in orthotopic and ectopic RG-2 gliomas, tumor tissue voxels were selected by identifying the respective tumors on the $T_{2}$ weighted anatomical scans in addition to the pattern of positive contrast enhancement within the tumor tissue extravascular space on one of the 2 minute high FA dynamic scan data sets acquired between 175 and 225 minutes, since this was the time frame of maximal contrast enhancement within the tumor tissue extravascular space for Gd-G5, Gd-G6, and Gd-G7 dendrimer animal groups. For the Gd-G8 animal group, although there was no significant positive contrast enhancement within the tumor tissue extravascular space on the dynamic scan data sets, the outline of the positive contrast enhancement within the tumor microvasculature on one of the dynamic scan data sets acquired between 175 and 225 minutes was sufficient to identify tumor tissue. The selected orthotopic and ectopic RG-2 glioma tumor tissue voxels represented the respective whole tumor volumes. To determine the change in Gd concentration over time, the whole tumor volumes were then identified on the co-registered high FA dynamic scan data sets of the other time points. The average whole tumor Gd concentration values were then calculated for each time point.

For each Gd-dendrimer generation, the average Gd concentrations obtained from the common carotid arteries, the orthotopic RG-2 glioma, and the ectopic RG-2 glioma were plotted over time using Matlab (Version 7.1; The MathWorks Inc, Natick, MA). The pharmacokinetics of Gd-dendrimers in blood were qualitatively assessed due to limited number of voxels available from the common carotid artery for analysis in the context of the known limitations of dynamic contrast-enhanced MRI-based acquisition of arterial input functions.

It was possible to quantify the pharmacokinetics of Gddendrimer generations in tumor tissues over 600 to 700 minutes. Best fit curves were calculated using the Matlab Curve Fitting Toolbox (Version 1.1.4; The MathWorks Inc) using a bi-exponential function (Eq. 4).

$$
[G d]_{t}=a e^{b t}+c e^{d t}
$$

where

$[G d]_{t}=$ predictive $G d$ concentration at time $t \min (\mathrm{mM})$

$a(\mathrm{mM}), b\left(\mathrm{~min}^{-1}\right), c(\mathrm{mM}), d\left(\mathrm{~min}^{-1}\right)=$ parameters to be determined for best fit

The first term, $a e^{b t}$, represents the fast initial exponential rise in Gd concentration and the second term, $c e^{d t}$, repre- 
sents the slow subsequent exponential decay in Gd concentration over time. The 95\% confidence intervals (CI) and the root mean squared errors (RMSE) for the orthotopic and ectopic RG-2 glioma Gd concentration curve profiles were calculated.

\section{Results \\ Physical properties of naked PAMAM and Gd-PAMAM dendrimer generations}

The physical properties of naked PAMAM dendrimers (Starburst G5-G8, ethylenediamine core; Sigma-Aldrich, St. Louis, MO) and Gd-DTPA functionalized PAMAM dendrimers were characterized. Within each dendrimer generation, the amount of increase in the molecular weight between the naked dendrimer and the functionalized dendrimer is proportional to the percent conjugation of Gd-DTPA (Table 1). For each successively higher dendrimer generation, the percent conjugation of Gd-DTPA is lower due to greater steric hindrance encountered in the chelation reaction process (Table 1 ). The Gd-dendrimer molar relaxivities, which are the constants of proportionality required for calculation of Gd concentration from Gd signal intensity, ranged between 9.81 and $10.051 / \mathrm{mM}^{*} \mathrm{~s}$ (Table 1).

ADF STEM of Gd-G5 through Gd-G8 dendrimers demonstrated uniformity in particle shape and size within any particular Gd-dendrimer generation (Figure 1). ADF STEM confirmed a small increase of approximately $2 \mathrm{~nm}$ in particle diameter between successive generations (Figure 1). The masses of Gd-G7 and Gd-G8 dendrimers were sufficient that the sizes and molecular weights of these Gd-dendrimer generations could be measured by ADF STEM and STEM-EFTEM, respectively. The molecular weights and diameters of one hundred Gd-G7 and Gd-G8 dendrimers were measured. The average molecular weight of Gd-G7 was $283 \pm 5 \mathrm{kDa}$ and that of Gd-G8 dendrimers was $490 \pm 5 \mathrm{kDa}$ (mean \pm standard error of the mean) (Table 1). The average diameter of Gd-G7 dendrimers was $10.9 \pm 0.7 \mathrm{~nm}$ and that of Gd-G8 dendrimers was $12.7 \pm$ $0.7 \mathrm{~nm}$ (mean \pm standard deviation).

\section{Permeability of the BTB of orthotopic and ectopic RG-2 gliomas to Gd-PAMAM dendrimer generations}

Gd-G5 dendrimers extravasated across the BTB of both orthotopic and ectopic RG-2 gliomas and accumulated within the respective tumor tissue extravascular spaces (Figure 2, panels A and E). However, the Gd-G5 dendrimers extravasated to a lesser extent across the BTB of orthotopic RG-2 gliomas than the BTB of ectopic RG-2 gliomas indicating the BTB of orthotopic RG-2 gliomas was less permeable than the BTB of ectopic RG-2 gliomas. Thus, the peak Gd concentration of Gd-G5 dendrimers in orthotopic tumors was $0.147 \mathrm{mM}$, whereas the peak Gd concentration of Gd-G5 dendrimers in ectopic tumors was $0.195 \mathrm{mM}$ (Table 2, Additional file 1).

Gd-G6 dendrimers also extravasated across the BTB of both orthotopic and ectopic RG-2 gliomas and accumulated within the respective tumor tissue extravascular spaces (Figure 2, panels B and F). Gd-G6 dendrimers accumulated to lesser extent than Gd-G5 dendrimers in both orthotopic and ectopic tumor tissue extravascular spaces. As was the case for Gd-G5 dendrimers, the Gd-G6 dendrimers extravasated to a lesser extent across the BTB of orthotopic RG-2 gliomas than the BTB of ectopic RG-2 gliomas, once again indicating the BTB of orthotopic RG-2 gliomas was less permeable than the BTB of ectopic RG-2 gliomas. Thus, the peak Gd concentration of Gd-G6 dendrimers in orthotopic tumors was $0.106 \mathrm{mM}$, whereas the peak Gd concentration of Gd-G6 dendrimers in ectopic tumors was $0.144 \mathrm{mM}$.

Gd-G7 dendrimers minimally extravasated across the BTB of both orthotopic and ectopic RG-2 gliomas and so minimally accumulated within the respective tumor tissue extravascular spaces (Figure 2, panels C and G). Gd-G7 dendrimers accumulated to an even lesser extent than Gd-G6 dendrimers in both orthotopic and ectopic tumor tissue extravascular spaces. As was the case for Gd-G6 dendrimers, the Gd-G7 dendrimers extravasated to a lesser extent across the BTB of orthotopic RG-2 gliomas than the BTB of ectopic RG-2 gliomas, once again indicating the BTB of orthotopic RG-2 glio-

Table I: Physical properties of PAMAM and Gd-PAMAM dendrimers

\begin{tabular}{cccccc}
\hline $\begin{array}{c}\text { Dendrimer generation } \\
(\mathrm{G})\end{array}$ & Terminal amines $(\#)$ & $\begin{array}{c}\text { Naked PAMAM } \\
\text { molecular weight \# } \\
(\mathrm{kDa})\end{array}$ & $\begin{array}{c}\text { Gd-PAMAM dendrimer } \\
\text { molecular weight }(\mathrm{kDa})\end{array}$ & $\begin{array}{c}\text { Gd-DTPA conjugation } \\
(\%)\end{array}$ & $\begin{array}{c}\text { Molar relaxivity\& } \\
(\mathrm{I} / \mathrm{mM} * \mathrm{~s})\end{array}$ \\
\hline G5 & 128 & 29 & $79 \dagger$ & 52 & 9.81 \\
G6 & 256 & 58 & $138 \dagger$ & 45 & 10.04 \\
G7 & 512 & 116 & $283 \ddagger$ & 43 & 9.82 \\
G8 & 1024 & 233 & $490 \ddagger$ & 36 & 10.05 \\
\hline
\end{tabular}

\#molecular weight obtained from Dendritech, Inc. tmolecular weight measured by MALDI TOF MS

fmean molecular weight measured by ADF STEM and EFTEM

\&molar relaxivity of Gd-DTPA measured to be $4.131 / \mathrm{mM}^{*} \mathrm{~s}$ 

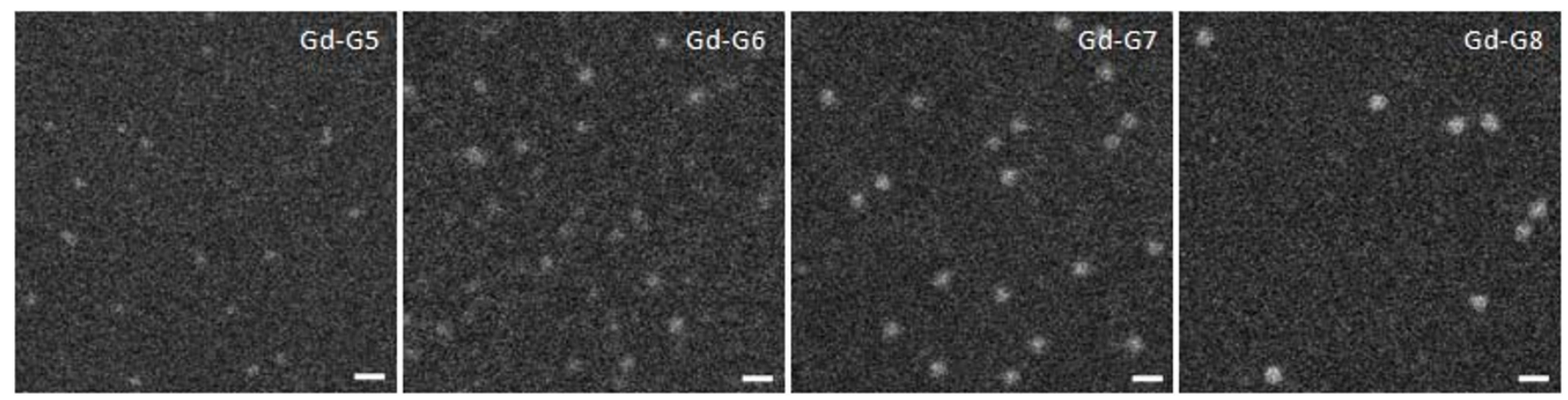

Figure I

Transmission electron microscopy of higher generation Gd-dendrimers. Annular dark-field scanning transmission electron microscopy (ADF STEM) images of unstained Gd-G5, Gd-G6, Gd-G7, and Gd-G8 dendrimers adsorbed onto an ultrathin carbon support film. The diameters of one hundred Gd-G7 and Gd-G8 dendrimers were measured. Scale bar $=20$ nm.

mas was less permeable than the BTB of ectopic RG-2 gliomas. Thus, the peak Gd concentration of Gd-G7 dendrimers in orthotopic tumors was $0.064 \mathrm{mM}$, whereas the peak Gd concentration of Gd-G7 dendrimers in ectopic tumors was $0.084 \mathrm{mM}$ (Table 2, Additional file 1).

Gd-G8 dendrimers did not extravasate across the BTB of orthotopic and ectopic RG-2 gliomas. The change in Gd concentration over time for both orthotopic and ectopic RG-2 gliomas was similar (Figure 2, panels D and H). The peak Gd concentrations of Gd-G8 dendrimers in both orthotopic and ectopic tumors were similar: the peak Gd concentration of Gd-G8 dendrimers in orthotopic tumors was $0.049 \mathrm{mM}$ and that in ectopic tumors was $0.052 \mathrm{mM}$ (Table 2, Additional file 1). The peak Gd concentrations in orthotopic and ectopic tumors reflect the peak Gd-G8 dendrimer concentrations within the microvasculature of the respective tumors and not the extravascular tumor tissue space.

\section{Physiologic upper limit of pore size within the BTB of orthotopic and ectopic RG-2 gliomas as visualized on Gd concentration maps}

For each of the Gd-dendrimer generations, after the initial 15 minute dynamic scan, the orthotopic and ectopic RG2 gliomas of one additional animal were imaged every 10 minutes for a total of 175 minutes, while the animal was under continuous anesthesia. The Gd concentration maps from selected dynamic scans of these imaging sessions are shown in Figure 3. The hemodynamic depression associated with the continuous anesthesia is reflected in the lower peak contrast enhancement observed.

Gd-G5 dendrimers readily extravasated across the BTB of both orthotopic and ectopic RG-2 gliomas and accumulated over time within the respective tumor tissue extravascular spaces, as evidenced by the significant positive contrast enhancement over time in the respective tumor tissues (Figure 3, first row). Gd-G6 dendrimers also extravasated across the BTB of both orthotopic and ectopic RG-2 gliomas and accumulated over time within the respective tumor tissue extravascular spaces (Figure 3, second row), although to a lesser extent than Gd-G5 dendrimers (Figure 3, first row).

Gd-G7 dendrimers minimally extravasated across the BTB of both orthotopic and ectopic RG-2 gliomas and so minimally accumulated over time within the respective tumor tissue extravascular spaces (Figure 3, third row). Gd-G8 dendrimers did not extravasate over time across the BTB of both orthotopic and ectopic RG-2 gliomas, but instead

Table 2: Gd-PAMAM dendrimer peak concentrations in orthotopic RG-2 gliomas versus ectopic RG-2 gliomas*

\begin{tabular}{ccccc}
\hline $\begin{array}{c}\text { Gd-dendrimer generation } \\
(\mathrm{G})\end{array}$ & $\begin{array}{c}\text { Peak concentration in } \\
\text { orthotopic RG-2 gliomas } \\
(\mathrm{mM})\end{array}$ & $\begin{array}{c}\text { Peak concentration time } \\
\text { point (min) }\end{array}$ & $\begin{array}{c}\text { Peak concentration in } \\
\text { ectopic RG-2 gliomas (mM) }\end{array}$ & $\begin{array}{c}\text { Peak concentration time } \\
\text { point (min) }\end{array}$ \\
\hline Gd-G5 & 0.147 & 167 & 0.195 & 149 \\
Gd-G6 & 0.106 & 200 & 0.144 & 189 \\
Gd-G7 & 0.064 & 75 & 0.084 & 107 \\
Gd-G8 & 0.049 & 77 & 0.052 & 81
\end{tabular}

$* 95 \%$ confidence intervals $(\mathrm{Cl})$ and root mean squared errors (RMSE) for best fit curve concentrations from the bi-exponential function $[\mathrm{Gd}]_{t}=$ $a e^{b t}+c e^{d t}$ are reported in Additional file I 

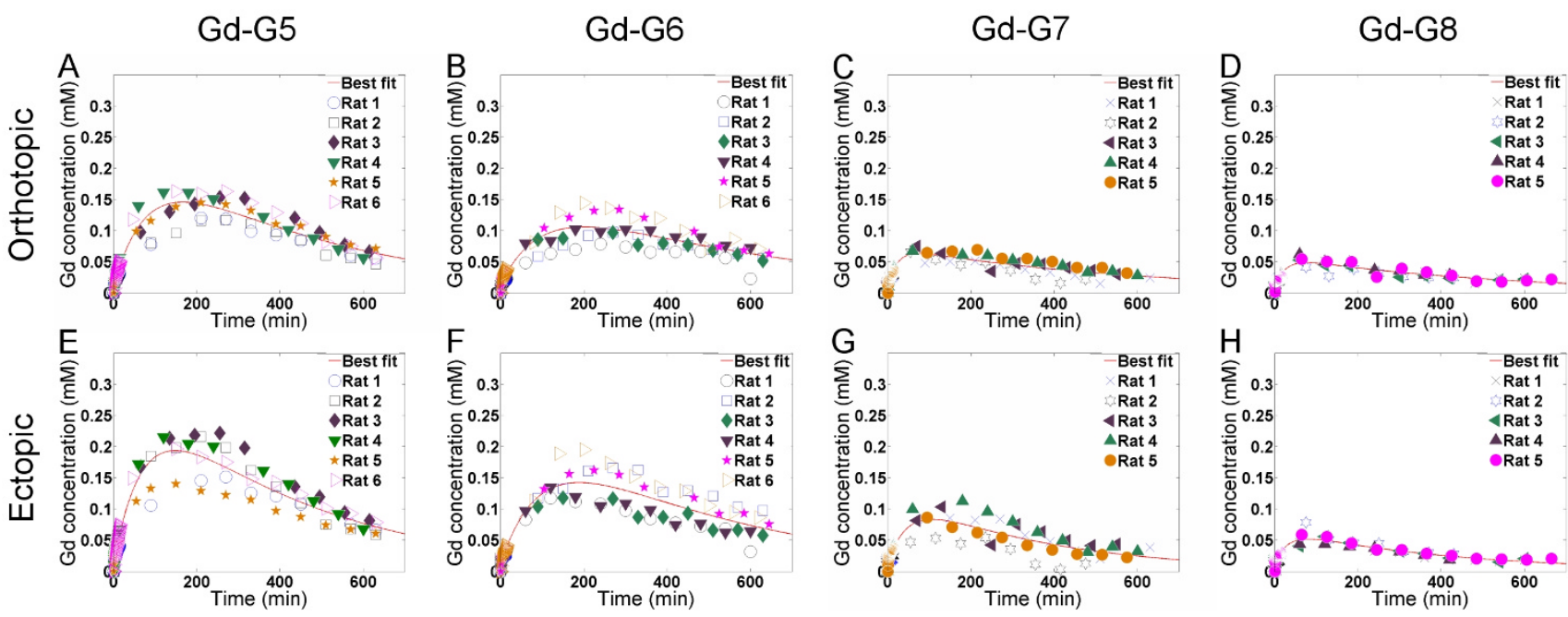

Figure 2

Pharmacokinetics of Gd-dendrimer generations in orthotopic RG-2 gliomas and ectopic RG-2 gliomas over $\mathbf{6 0 0}$ to $\mathbf{7 0 0}$ minutes. Respective Gd-dendrimer generation was intravenously infused over I minute $(0.09 \mathrm{mmol} \mathrm{Gd} / \mathrm{kg}) \mathrm{dur}-$ ing the initial 15 minute dynamic contrast-enhanced MRI scan session. Subsequent dynamic scan sessions of re-anesthetized animals were conducted at 30 to 90 minute time intervals. Whole tumor tissue Gd concentrations for the orthotopic and ectopic RG-2 gliomas were calculated for each of the dynamic scan session time points. Shown is the change in the Gd concentration of respective Gd-dendrimer generations in orthotopic RG-2 gliomas and ectopic RG-2 gliomas over 600 to 700 minutes. Superimposed is the best fit curve Gd concentration curve for the respective Gd-dendrimer generations. Panels $A$ through $D$ are orthotopic glioma Gd concentrations over time. Panels $E$ through $\mathrm{H}$ are ectopic glioma Gd concentrations over time $A$. Gd-G5 (Orthotopic, N = 6), B. Gd-G6 (Orthotopic, N = 6), C. Gd-G7 (Orthotopic, N = 5), D. Gd-G8 (Orthotopic, N = 5), E. Gd-G5 (Ectopic, N = 6), F. Gd-G6 (Ectopic, $N=6$ ), G. Gd-G7 (Ectopic, $N=5$ ), H. Gd-G8 (Ectopic, $N=5$ ).

remained within the tumor microvasculature, as evidenced by the lack of contrast enhancement over time within the respective tumor tissue extravascular spaces (Figure 3, fourth row). Therefore, the physiologic upper limit of pore size within the BTB of both malignant brain tumors and peripheral solid tumors is equivalent. Since the diameter of our Gd-G7 dendrimers and Gd-G8 dendrimers was $10.9 \pm 0.7 \mathrm{~nm}$ and $12.7 \pm 0.7 \mathrm{~nm}$ (mean \pm standard deviation), the upper limit of pore size within the BTB of both orthotopic RG-2 gliomas and ectopic RG2 gliomas is approximately $12 \mathrm{~nm}$.

\section{Discussion}

In the BTB of malignant solid tumor microvasculature, the anatomic pore sizes of trans-endothelial cell fenestrations, caveolae and VVOs range between $40 \mathrm{~nm}$ to 200 $\mathrm{nm}[10,13,14]$, and the sizes of inter-endothelial cell gaps range between $100 \mathrm{~nm}$ and $4700 \mathrm{~nm}[10,12,13]$. Irrespective of tumor host site, trans-endothelial cell fenestrations, caveolae, and VVOs are present more often than the inter-endothelial cell gaps in the BTB of malignant solid tumors $[4,9,10]$. Due to host site influence the BTB of peripheral tumors has more frequent trans-endothelial cell fenestrations, caveolae and VVOs, and larger interendothelial cell gaps than the BTB of malignant brain tumor microvasculature $[6,10]$. The higher permeability of the BTB of peripheral tumors than that of brain tumors has been attributed to the larger anatomic pore sizes of the inter-endothelial cell gaps $[12,15]$. We reasoned that in the physiologic state in vivo the intact luminal glycocalyx layer would be the primary impediment to the transvascular passage of even small nanoparticles across the BTB of malignant solid tumors independent of tumor host site.

In this study, with dynamic contrast-enhanced MRI we imaged the blood and tumor tissue pharmacokinetics of intravenously infused Gd-PAMAM dendrimer nanoparticles G5 through G8 over 600 to 700 minutes. We compared the permeability of the BTB of RG-2 gliomas grown within the brain, the orthotopic site, to that of the BTB of RG-2 gliomas grown outside the brain in the temporalis skeletal muscle, the ectopic site. We used this animal model to characterize the differences in the permeability of the BTB of a malignant brain tumor to that of the BTB of a peripheral solid tumor, and to define the upper limit of pore size within the BTB of the respective solid tumors. Using this approach, we found that the physiologic upper limit of pore size in the BTB of brain RG-2 gliomas and peripheral RG-2 gliomas is approximately $12 \mathrm{~nm}$.

In the case of brain RG-2 gliomas, we report here that the physiologic upper limit of pore size in the BTB of ortho- 


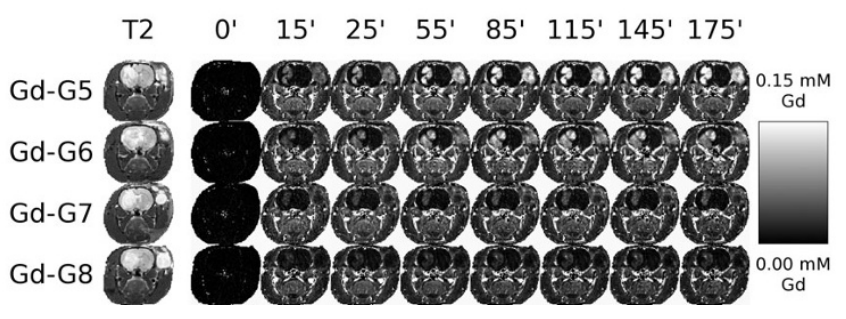

Figure 3

Gd concentration maps of $\mathbf{G d}$-dendrimer contrast enhancement over I 75 minutes. For one additional ani$\mathrm{mal}$ in each $\mathrm{Gd}$-dendrimer generation group the respective Gd-dendrimer generation was intravenously infused over I minute $(0.09 \mathrm{mmol} \mathrm{Gd} / \mathrm{kg})$ while the animal was maintained under anesthesia for the duration of the I 75 minute dynamic contrast-enhanced MRI session. Voxel-by-voxel Gd concentration maps were generated. Shown are the voxel-by-voxel Gd concentration maps for the respective Gd-dendrimer generations at the 15 minute time point and then at 30 minute time intervals thereafter. First row, Gd-G5 dendrimer (Orthotopic RG-2 glioma tumor volume, $45 \mathrm{~mm}^{3}$; ectopic RG-2 glioma tumor volume, II $3 \mathrm{~mm}^{3}$ ). Second row, Gd-G6 dendrimer (Orthotopic RG-2 glioma tumor volume, $97 \mathrm{~mm}^{3}$; ectopic RG-2 glioma tumor volume, $184 \mathrm{~mm}^{3}$ ). Third row, Gd-G7 dendrimer (Orthotopic RG-2 glioma tumor volume, $53 \mathrm{~mm}^{3}$; ectopic RG-2 glioma tumor volume, $135 \mathrm{~mm}^{3}$ ). Fourth row, Gd-G8 dendrimer (Orthotopic RG-2 glioma tumor volume, $50 \mathrm{~mm}^{3}$; ectopic RG-2 glioma tumor volume, $163 \mathrm{~mm}^{3}$ ).

topic RG-2 gliomas growing in brain tissue is approximately $12 \mathrm{~nm}$. Our present finding is in agreement with our previously reported finding that the upper limit of pore size in the BTB of orthotopic RG-2 gliomas is approximately $12 \mathrm{~nm}[22]$. Both in our prior and present work, we probed the upper limit of the pore size within the BTB with dynamic contrast-enhanced MRI using successively higher generation Gd-DTPA labeled PAMAM dendrimer nanoparticles with a neutralized particle exterior. The positive charge on exterior of the naked PAMAM dendrimer generations was neutralized by the conjugation of GdDTPA (charge -2) to approximately $40 \%$ to $50 \%$ of the terminal amines on the exterior. Therefore, the Gd-DTPA labeled dendrimer generations that were used for this study would have not been toxic to the negatively charged glycocalyx overlaying the endothelial cells of the BTB.

In the case of peripheral RG-2 gliomas, we report here that the physiologic upper limit of pore size in the BTB of ectopic RG-2 gliomas growing in skeletal muscle is equivalent to the upper limit of pore size in the BTB of orthotopic RG-2 gliomas growing in brain tissue, and is also approximately $12 \mathrm{~nm}$. The physiologic upper limit of pore size in the BTB of peripheral RG-2 gliomas that we report here is significantly lower than what has been previously reported[15]. In the past, the physiologic upper limit of the pore size within the BTB of orthotopic and ectopic malignant peripheral tumors has been probed by intravital fluorescence microscopy 24 hours after the intravenous infusion of liposomes and microspheres with a cationic exterior, and it has been reported the upper limit of the pore size within the BTB of peripheral tumors is between $200 \mathrm{~nm}$ and $1200 \mathrm{~nm}[15]$. This higher upper limit of pore size would be most likely due to the toxicity of the cationic liposomes and microspheres to the negatively charged glycocalyx overlaying the endothelial cells of the BTB. The circulation of cationic particles for 24 hours would be sufficient time to expose the underlying smaller-sized trans-endothelial cell fenestrations and WOs as well as the larger-sized inter-endothelial cell gaps. The transvascular extravasation of the particles across the exposed inter-endothelial cell gaps into the tumor tissue extravascular space, or alternatively, entrapment in the peri-vascular space along the basement membrane would result in the over-estimation of the actual physiologic upper limit of pore size within the BTB.

We found that Gd-G5, Gd-G6, and Gd-G7 dendrimers extravasated across the BTB of ectopic RG-2 gliomas as well as that of orthotopic RG-2 gliomas. However, these Gd-dendrimer generations extravasated to a greater extent across the BTB of ectopic RG-2 gliomas than the BTB of orthotopic RG-2 gliomas, as Gd-G5, Gd-G6, and Gd-G7 dendrimers achieved higher peak concentrations in the tumor tissue extravascular space of ectopic RG-2 malignant gliomas than in the tumor tissue extravascular space of orthotopic RG-2 malignant gliomas. Based on these findings, the BTB of the ectopic RG-2 malignant gliomas is more permeable than the BTB of orthotopic RG-2 malignant gliomas. The observed higher permeability of the BTB of ectopic RG-2 gliomas in this animal model may be in part due to host site dependent differences in tumor volume, since the tumor volumes of the ectopic RG-2 gliomas where generally larger than those of the orthotopic RG-2 gliomas (Figure 4). Although this may be the case, the higher permeability of BTB of ectopic RG-2 gliomas compared to that of the BTB of orthotopic RG-2 gliomas is consistent with the reported higher permeability of the BTB of malignant peripheral tumors compared to that of the BTB of malignant brain tumors[5,7].

With each successively higher Gd-dendrimer generation there was an approximately $2 \mathrm{~nm}$ increase in Gd-dendrimer diameter. Although there were relatively small increases in Gd-dendrimer particle sizes, there were significant decreases in particle extravasation across the BTB with increasing Gd-dendrimer generation, irrespective of RG-2 glioma host site. Gd-G7 dendrimers extravasated only minimally across the BTB, and the Gd-G8 dendrim- 


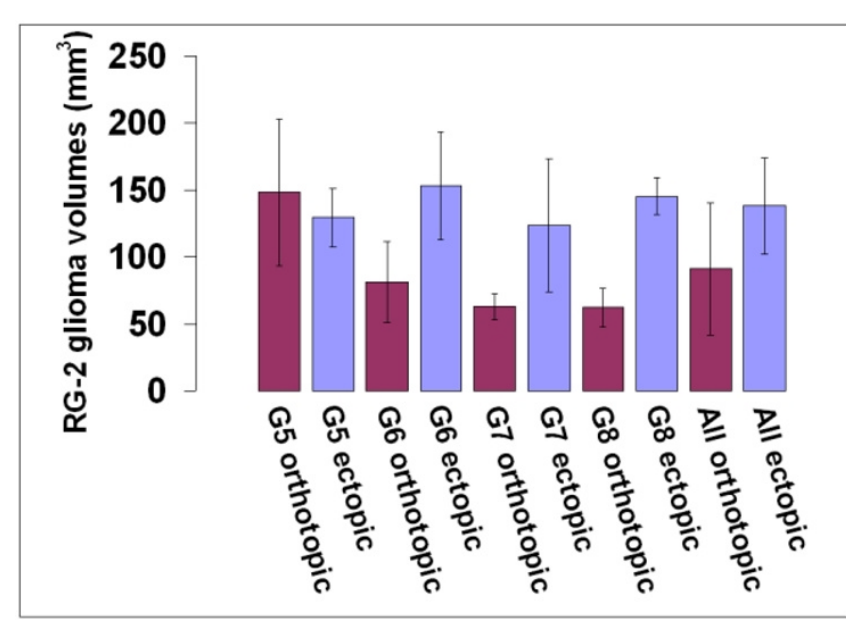

Figure 4

Tumor volumes of orthotopic and ectopic RG-2 gliomas of each Gd-dendrimer generation. Whole tumor tissue volumes, in $\mathrm{mm}^{3}$, were determined for the orthotopic and ectopic RG-2 gliomas of each of the Gd-dendrimer generation groups using the $T_{2}$ weighted anatomical scans and dynamic contrast-enhanced MRI data sets as described in the Methods section. Shown are the average whole tumor volumes of orthotopic and ectopic RG-2 gliomas of each Gddendrimer generation. A. Gd-G5 (Orthotopic, $N=6$;

Ectopic, $N=6$ ), B. Gd-G6 (Orthotopic, $N=6$; Ectopic, $N=$ 6), C. Gd-G7 (Orthotopic, $N=5$; Ectopic, $N=5$ ), D. Gd-G8 (Orthotopic, $N=5$; Ectopic, $N=5$ ). Error bars represent standard deviation.

ers were large enough that these particles did not extravasate across either the BTB of ectopic RG-2 gliomas or that of orthotopic RG-2 gliomas. As a result, Gd-G8 dendrimers did not accumulate over time in the respective tumor tissue extravascular spaces, and instead remained in the tumor microvasculature. The peak Gd concentrations of Gd-G8 dendrimers in ectopic RG-2 gliomas and orthotopic RG-2 gliomas were similar and reflect the peak Gd-
G8 dendrimer concentrations within the microvasculature of the respective tumors.

We found that the blood half-lives of Gd-G5 and Gd-G6 dendrimers to be longer than those of Gd-G7 and Gd-G8 dendrimers (Figure 5). In case of Gd-G5 and Gd-G6 dendrimers, the relatively longer blood half-lives are due to the sizes of these Gd-dendrimer generations being large enough to evade kidney filtration following transvascular extravasation across the discontinuous microvasculature of the glomeruli of the kidneys[30], yet small enough to evade liver and spleen reticuloendothelial system opsonization following transvascular extravasation across the discontinuous microvasculature of the liver and spleen[31]. Therefore, Gd-G5 and Gd-G6 dendrimers were not effectively cleared from blood circulation and had longer blood half-lives than Gd-G7 and Gd-G8 dendrimers. In the case of Gd-G7 and Gd-G8 dendrimers, due to the relatively few number of voxels available for analysis and the finite sensitivity of dynamic contrast-enhanced MRI-based analysis, it was not possible to accurately detect the relatively small changes in blood Gd concentration at the latter imaging time points when the Gd-G7 and Gd-G8 dendrimer generations had been cleared from the blood circulation (Figure 5, panels $\mathrm{C}$ and D). However, it was possible to qualitatively assess the differences in the blood half-lives of Gd-G7 and Gd-G8 dendrimers compared to those of the Gd-G5 and Gd-G6 dendrimers. The blood half-lives of Gd-G7 and Gd-G8 dendrimers were shorter than those of the Gd-G5 and Gd-G6 dendrimers likely due to the sizes of these Gd-dendrimers being too large to evade opsonization by reticuloendothelial system of the liver and spleen[31]. Even though Gd-G7 dendrimers were small enough to extravasate across the BTB and Gd-G8 dendrimers were too large to extravasate across the BTB, both Gd-G7 and Gd-G8 dendrimers were effectively cleared from blood circulation and had shorter blood half-lives than Gd-G5 and Gd-G6 dendrimers. These findings suggest that nanoparticles within the size range of
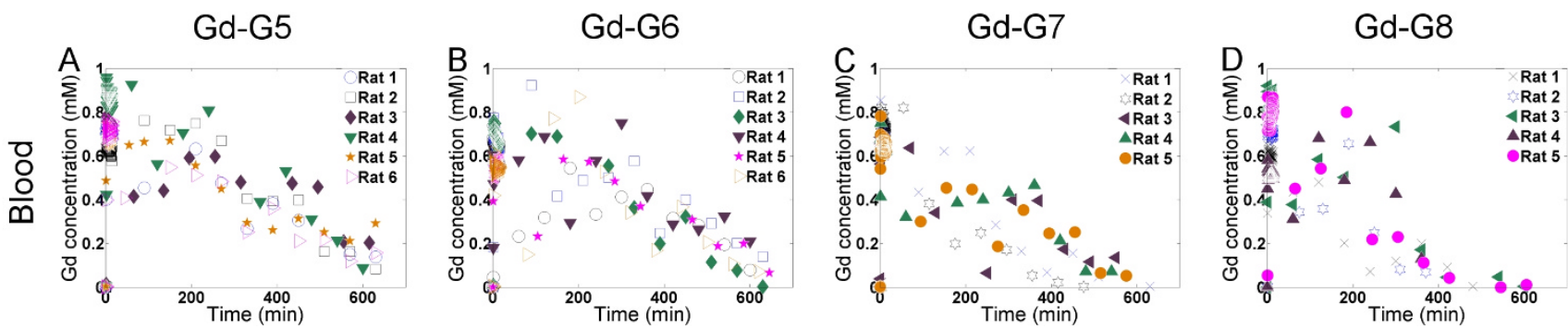

\section{Figure 5}

Blood pharmacokinetics of Gd-dendrimer generations over $\mathbf{6 0 0}$ to $\mathbf{7 0 0}$ minutes. Five to ten voxels were selected from within the common carotid arteries. For the selected voxels, the average blood Gd concentrations were determined for each of the dynamic scan session time points. Shown is the change in average blood Gd concentration of the respective Gddendrimer generations over 600 to 700 minutes. A. Gd-G5 (N=6), B. Gd-G6 (N = 6), C. Gd-G7 (N= 5), D. Gd-G8 (N = 5). 
Gd-G5 and Gd-G6 dendrimers would be both permeable to the BTB of malignant solid tumor microvasculature and also possess blood half-lives sufficiently long to allow for particles to effectively accumulate over time within the tumor tissue extravascular space by the enhanced permeation and retention (EPR) effect[32].

Since the sizes of hydrated dendrimer generations, measured by small-angle X-ray scattering (SAXS)[33] and small-angle neutron scattering (SANS)[34], are similar to the sizes of respective dehydrated and stained dendrimer generations measured by TEM[35], here we used ADF STEM to the measure the sizes of the Gd-G7 dendrimers and Gd-G8 dendrimers dried on ultrathin carbon support film $[24,25]$. We found the diameters of the Gd-G7 dendrimers to be $10.9 \pm 0.7 \mathrm{~nm}$ and those of the Gd-G8 dendrimers to be $12.7 \pm 0.7 \mathrm{~nm}$ (mean \pm standard deviation). Since Gd-G7 dendrimers were permeable to both the BTB of ectopic RG-2 gliomas and orthotopic RG-2 gliomas, but the Gd-G8 dendrimers were not, this establishes the effective physiologic upper limit of pore size in both the BTB of ectopic RG-2 gliomas and orthotopic RG-2 gliomas as being approximately $12 \mathrm{~nm}$.

The previously reported higher physiologic upper limit of pore size in the BTB of malignant solid tumors, based on intra-vital fluorescence microscopy of tumor tissue 24 hours following the intravenous infusion of cationic nanoparticles, appears to have been a gross over-estimation of the actual physiologic upper limit of pore size. The most plausible explanation for this is that the positively charged exterior of the cationic nanoparticles was toxic to the negatively charged glycocalyx surface coat of the BTB. We report here, based on dynamic contrast-enhanced MRI of tumor tissue following the intravenous infusion of neutralized nanoparticles, that the physiologic upper limit of pore size is much lower, being approximately $12 \mathrm{~nm}$, when the luminal fibrous glycocalyx of the BTB is maintained intact.

The ultrastructure of the glycocalyx has been previously investigated in frog mesentery capillaries since the morphology of this type of microvasculature is similar to that of mammalian microvasculature of the continuous type, for example that of skeletal muscle[36,37]. In such continuous microvasculature, there are small pores in the endothelial barrier underlying the glycocalyx that allow for the minimal transvascular extravasation of macromolecules smaller than 4 to $5 \mathrm{~nm}$ in diameter across the barrier[38,39]. It has been reported that when the fibrous meshwork of the glycocalyx layer overlaying these small pores is enzymatically degraded, then there is an increase in the transvascular extravasation of macromolecules across the endothelial barrier[40,41] even though there are no accompanying anatomic changes in the underlying pores[41]. Based on such work, it would be reasonable to speculate that the observed increase in transvascular extravasation of macromolecules across the endothelial barrier of continuous microvasculature is a result of an increase in the physiologic upper limit of pore size in the barrier due to the disruption of the glycocalyx layer. The damage that occurs to the glycocalyx of the endothelial barrier of continuous microvasculature following enzymatic degradation would be analogous to that which occurs to the glycocalyx of the BTB of malignant tumor microvasculature following prolonged exposure to the positive exterior of cationic particles.

In the case of the BTB of malignant solid tumor microvasculature, we report here that in the physiologic state in vivo that only particles smaller than approximately $12 \mathrm{~nm}$ in diameter can effectively extravasate across the BTB independent of tumor location. Although we found that the physiologic upper limit of pore size in the BTB of brain tumors (orthotopic RG-2 gliomas) as well as peripheral tumors (ectopic RG-2 gliomas) was equivalent, the transvascular extravasation of the permeable particles (i.e. GdG5, Gd-G6, and Gd-G7 dendrimers) was greater across the BTB of the peripheral tumors. Even though in this work we did not study the ultrastructure of the glycocalyx of the BTB of brain and peripheral tumor microvasculature, we suspect that there are similarities in the arrangement and spacing of the glycocalyx fibers overlaying the pores within the BTB of brain and peripheral tumor microvasculature. This would account for the physiologic upper limit of pore size in the BTB of malignant solid tumor microvasculature being equivalent and independent of tumor location. The higher permeability of the BTB of malignant peripheral tumors to macromolecules, in this case the GdG5, Gd-G6 and Gd-G7 dendrimer nanoparticles, may then be explained by the presence of more pores underlying the glycocalyx, which would allow for the transvascular extravasation of greater numbers of particles smaller than approximately $12 \mathrm{~nm}$ in diameter.

\section{Conclusion}

We report here that the physiologic upper limit of pore size in the BTB of malignant solid tumor microvasculature is approximately 12 nanometers. Since in the physiologic state in vivo the fibrous glycocalyx overlays the luminal surface of the BTB of both brain tumor and peripheral tumor microvasculature, the physiologic upper limit of pore size in the BTB of malignant solid tumor microvasculature is equivalent and independent of tumor host site. The higher permeability of malignant peripheral tumor microvasculature to macromolecules smaller than approximately $12 \mathrm{~nm}$ in diameter is attributable to the presence of a greater number of pores underlying the glycocalyx of the BTB of peripheral tumor microvasculature. 


\section{Competing interests}

The authors declare that they have no competing interests.

\section{Authors' contributions}

HS conceptualized and designed overall study; performed MRI experiments, analyzed MRI data, interpreted overall study results, and wrote the manuscript. ASK assisted with MRI experiments, data analysis, and figure preparation. HW synthesized functionalized dendrimers. AAS characterized functionalized dendrimers with electron microscopy. CMW assisted with functionalized dendrimer synthesis. MAA assisted with electron microscopic dendrimer characterization. GLG supervised synthesis of the functionalized dendrimers. RDL supervised characterization of functionalized dendrimers with electron microscopy. HV assisted with MRI experiments, data analysis, and figure preparation. All authors read and proofed the final manuscript.

\section{Additional material}

\section{Additional file 1}

95\% confidence intervals (CI) and root mean squared errors (RMSE) for best fit curve concentrations from the bi-exponential function $[\mathrm{Gd}]_{\mathrm{t}}=\mathbf{a e}^{\mathrm{bt}}+\mathbf{c e}^{\mathrm{dt}}$. The data in the table represent the statistical analysis for the orthotopic and ectopic RG-2 glioma Gd concentration curve profiles for the respective Gd-dendrimer generations over 600 to 700 minutes. A best fit was established for each Gd concentration curve profile as indicated by the corresponding low RMSE value. Note: 1 RMSE per profile. Click here for file

[http://www.biomedcentral.com/content/supplementary/14795876-7-51-S1.doc]

\section{Acknowledgements}

This study was funded by the National Institute of Biomedical Imaging and Bioengineering (NIBIB), and the Radiology and Imaging Sciences Program (CC).

\section{References}

I. Jain RK: Transport of molecules across tumor vasculature. Cancer Metastasis Rev 1987, 6:559-593.

2. Michel CC: Transport of macromolecules through microvascular walls. Cardiovascular Research 1996, 32:644-653.

3. Senger DR, Perruzzi CA, Feder J, Dvorak HF: A highly conserved vascular permeability factor secreted by a variety of human and rodent tumor cell lines. Cancer Research 1986, 46:5629-5632.

4. Roberts WG, Palade GE: Neovasculature induced by vascular endothelial growth factor is fenestrated. Cancer Res 1997, 57:765-772.

5. Monsky WL, Carreira CM, Tsuzuki Y, Gohongi T, Fukumura D, Jain RK: Role of host microenvironment in angiogenesis and microvascular functions in human breast cancer xenografts: Mammary fat pad versus cranial tumors. Clinical Cancer Research 2002, 8:1008-1013.

6. Roberts WG, Delaat J, Nagane M, Huang S, Cavenee WK, Palade GE: Host microvasculature influence on tumor vascular morphology and endothelial gene expression. American Journal of Pathology 1998, 153:1239-1248.

7. Hasegawa H, Ushio Y, Hayakawa T: Changes of the blood-brain barrier in experimental metastatic brain tumors. Journal of Neurosurgery 1983, 59:304-310.
8. Molnar P, Blasberg RG, Horowitz M: Regional blood-to-tissue transport in RT-9 brain tumors. Journal of Neurosurgery 1983, 58:874-884.

9. Feng D, Nagy JA, Dvorak AM, Dvorak HF: Different Pathways of Macromolecule Extravasation from Hyperpermeable Tumor Vessels. Microvascular Research 2000, 59:24-37.

10. Vick NA, Bigner DD: Microvascular abnormalities in virallyinduced canine brain tumors. Structural bases for altered blood-brain barrier function. J Neurol Sci 1972, I 7:29-39.

II. Feng D, Nagy JA, Dvorak HF, Dvorak AM: Ultrastructural studies define soluble macromolecular, particulate, and cellular transendothelial cell pathways in venules, lymphatic vessels, and tumor-associated microvessels in man and animals. Microscopy Research and Technique 2002, 57:289-326.

12. Hashizume H, Baluk P, Morikawa S, McLean JW, Thurston G, Roberge $S$, Jain RK, McDonald DM: Openings between defective endothelial cells explain tumor vessel leakiness. Am J Pathol 2000, I 56: I363-I380.

13. Schlageter KE, Molnar P, Lapin GD, Groothuis DR: Microvessel organization and structure in experimental brain tumors: Microvessel populations with distinctive structural and functional properties. Microvascular Research 1999, 58:3 I2-328.

14. Dvorak AM, Kohn S, Morgan ES, Fox P, Nagy JA, Dvorak HF: The vesiculo-vacuolar organelle (VVO): A distinct endothelial cell structure that provides a transcellular pathway for macromolecular extravasation. Journal of Leukocyte Biology 1996, 59:100-II5.

15. Hobbs SK, Monsky WL, Yuan F, Roberts WG, Griffith L, Torchilin VP, Jain RK: Regulation of transport pathways in tumor vessels: role of tumor type and microenvironment. Proc Natl Acad Sci USA 1998, 95:4607-46I2.

16. Lutty GA: The acute intravenous toxicity of biological stains, dyes, and other fluorescent substances. Toxicology and Applied Pharmacology 1978, 44:225-249.

17. Hardebo JE, Kahrstrom J: Endothelial negative surface charge areas and blood-brain barrier function. Acta Physiologica Scandinavica 1985, I 25:495-499.

18. Lockman PR, Koziara JM, Mumper RJ, Allen DD: Nanoparticle Surface Charges Alter Blood-Brain Barrier Integrity and Permeability. Journal of Drug Targeting 2004, I 2:635-64 I.

19. Pries AR, Secomb TW, Gaehtgens P: The endothelial surface layer. Pflugers Archiv European Journal of Physiology 2000, 440:653-666.

20. Campbell RB, Fukumura D, Brown EB, Mazzola LM, Izumi Y, Jain RK, Torchilin VP, Munn LL: Cationic charge determines the distribution of liposomes between the vascular and extravascular compartments of tumors. Cancer Research 2002, 62:683 I-6836.

21. Dellian M, Yuan F, Trubetskoy VS, Torchilin VP, Jain RK: Vascular permeability in a human tumour xenograft: Molecular charge dependence. British Journal of Cancer 2000, 82:15I3-I5I8.

22. Sarin H, Kanevsky AS, Wu H, Brimacombe KR, Fung SH, Sousa AA, Auh S, Wilson CM, Sharma K, Aronova MA, et al.: Effective transvascular delivery of nanoparticles across the blood-brain tumor barrier into malignant glioma cells. J Transl Med 2008, 6:80.

23. Weinbaum S, Zhang $X$, Han $Y$, Vink H, Cowin SC: Mechanotransduction and flow across the endothelial glycocalyx. Proceedings of the National Academy of Sciences of the United States of America 2003, 100:7988-7995.

24. Sousa AA, Leapman RD: Quantitative STEM mass measurement of biological macromolecules in a $300 \mathrm{kV}$ TEM. J Microsc 2007, 228:25-33.

25. Sousa AA, Aronova MA, Wu H, Sarin H, Griffiths GL, Leapman RD: Determining molecular mass distributions and compositions of functionalized dendrimer nanoparticles. Nanomedicine 2009 , 4:391-399.

26. Haacke EM, Brown RW, Thompson MR, Venkatesan M: Magnetic Resonance Imaging: Physical Principles and Sequence Design New York: Wiley; 1999.

27. Rohrer $M$, Bauer $H$, Mintorovitch J, Requardt M, Weinmann $H J$ Comparison of magnetic properties of MRI contrast media solutions at different magnetic field strengths. Invest Radiol 2005, 40:715-724.

28. Paxinos G, Watson C: The Rat Brain in Stereotaxic Coordinates. 4th edition. New York: Elsevier; 2004. 
29. Cox RW: AFNI: software for analysis and visualization of functional magnetic resonance neuroimages. Comput Biomed Res 1996, 29:162-173.

30. Soo Choi H, Liu W, Misra P, Tanaka E, Zimmer JP, Itty Ipe B, Bawendi MG, Frangioni JV: Renal clearance of quantum dots. Nat Biotechnol 2007, 25: I 165-1 I70.

31. Kaminskas LM, Boyd BJ, Karellas P, Henderson SA, Giannis MP, Krippner GY, Porter CJ: Impact of surface derivatization of poly-Llysine dendrimers with anionic arylsulfonate or succinate groups on intravenous pharmacokinetics and disposition. Mol Pharm 2007, 4:949-961.

32. Maeda H, Wu J, Sawa T, Matsumura Y, Hori K: Tumor vascular permeability and the EPR effect in macromolecular therapeutics: a review. J Control Release 2000, 65:27I-284.

33. Prosa T], Bauer B], Amis EJ, Tomalia DA, Scherrenberg R: A SAXS study of the internal structure of dendritic polymer systems. Journal of Polymer Science Part B: Polymer Physics 1997, 35:291 3-2924.

34. Nisato G, Ivkov R, Amis E): Size invariance of polyelectrolyte dendrimers. Macromolecules 2000, 33:4172-4176.

35. Jackson CL, Chanzy HD, Booy FP, Drake BJ, Tomalia DA, Bauer BJ, Amis E): Visualization of dendrimer molecules by transmission electron microscopy (TEM): Staining methods and cryoTEM of vitrified solutions. Macromolecules 1998, 31:6259-6265.

36. Mason JC, Curry FE, White IF, Michel CC: The ultrastructure of frog mesenteric capillaries of known filtration coefficient. $Q$ J Exp Physiol Cogn Med Sci. 1979, 64(3):2 17-224.

37. Bruns RR, Palade GE: Studies on blood capillaries. I. General organization of blood capillaries in muscle. Journal of Cell Biology 1968, 37:244-276.

38. Michel CC, Curry FE: Microvascular permeability. Physiological Reviews 1999, 79:703-76I.

39. Squire JM, Chew M, Nneji G, Neal C, Barry J, Michel C: Quasi-Periodic Substructure in the Microvessel Endothelial Glycocalyx: A Possible Explanation for Molecular Filtering? Journal of Structural Biology 200I, 136:239-255.

40. Henry CBS, Duling BR: Permeation of the luminal capillary glycocalyx is determined by hyaluronan. Am J Physiol. 1999, 277(2 Pt 2): $\mathrm{H} 508-\mathrm{H} 5 \mathrm{I} 4$.

4I. Adamson RH: Permeability of frog mesenteric capillaries after partial pronase digestion of the endothelial glycocalyx. Journal of Physiology 1990, 428: I-13.

Publish with Bio Med Central and every scientist can read your work free of charge

"BioMed Central will be the most significant development for disseminating the results of biomedical research in our lifetime. "

Sir Paul Nurse, Cancer Research UK

Your research papers will be:

- available free of charge to the entire biomedical community

- peer reviewed and published immediately upon acceptance

- cited in PubMed and archived on PubMed Central

- yours - you keep the copyright
BioMedcentral 